\title{
Assessing low-cost terrestrial laser scanners for deriving forest
}

\section{structure parameters}

\author{
Atticus E. L. Stovall 1,2 Jeff W. Atkins ${ }^{3}$ \\ 1 University of Maryland, College Park, MD, USA; atticus@umd.edu \\ 2 Biospheric Sciences Laboratory, NASA Goddard Space Flight Center, Greenbelt, MD, USA \\ 3 Center for Forest Assessment and Syntheses, USDA Forest Service Southern Research Station, New Ellen- \\ ton, SC, USA; jeffrey.atkins@usda.gov \\ * Correspondence: atticus@umd.edu
}

\begin{abstract}
The increasingly affordable price point of terrestrial laser scanners has led to a democratization of instrument availability, but the most common low-cost instruments have yet to be compared in terms of the consistency to measure forest structural attributes. Here, we compared two low-cost terrestrial laser scanners (TLS): the Leica BLK360 and the Faro Focus 120 3D. We evaluate the instruments in terms of point cloud quality, forest inventory estimates, tree-model reconstruction, and foliage profile reconstruction. Our direct comparison of the point clouds showed reduced noise in filtered Leica data. Tree diameter and height were consistent across instruments (4.4\% and $1.4 \%$ error, respectively). Volumetric tree models were less consistent across instruments, with $\sim 29 \%$ bias, depending on model reconstruction quality. In the process of comparing foliage profiles, we conducted a sensitivity analysis of factors affecting foliage profile estimates, showing a minimal effect from instrument maximum range (for forests less than $\sim 50 \mathrm{~m}$ in height) and surprisingly little impact from degraded scan resolution. Filtered unstructured TLS point clouds must be artificially re-gridded to provide accurate foliage profiles. The factors evaluated in this comparison point towards necessary considerations for future low-cost laser scanner development and application in detecting forest structural parameters.
\end{abstract}

Keywords: tls; tree; QSM; PAVD; foliage; sensor; lidar

\section{Introduction}

Since the early 2000s, terrestrial laser scanning (TLS) has matured into a robust, and tractable means of measuring forest structural attributes using light detection and ranging (LiDAR) from the ground [1]. TLS has been used to model branch architecture [2], quantify leaf angle distributions [3,4], assess habitat quality [5], estimate fuel loads [6-8], map forest microtopograpy $[9,10]$, examine biodiversity gradients $[11,12]$, and estimate forest biomass [13-18]. Forest biomass carbon storage and productivity and can be inferred from estimates of above-ground biomass $[19,20]$. However, uncertainties exist in the distribution of above-ground biomass, affecting the understanding terrestrial sink/source status [21]. Ground based measurements of forest structure are the current basis for all area-wide estimates of aboveground biomass [22]. Tree diameter and height are traditionally related to destructive measures of dry biomass and carbon to create allometric scaling relationships that can be applied across forest plots [23-25]. Allometric relationships are difficult to create and as a result, tend to be low in sample size, spatially biased, and often exclude large trees [15,26]. An accurate and unbiased method of measuring forest structure through more direct ground-based measurements would constrain uncertainty in forest carbon storage and productivity $[27,28]$.

TLS offers non-destructive means of estimating above-ground biomass [14,16], forest structure [29,30], canopy complexity [31], and structural traits [32], but much of the research employing TLS technology has used high-end TLS units costing more than $\$ 75,000$ 
USD--a price point directly limiting the widespread adoption of TLS methods. Until recently, high-end TLS units were the only commercially available units. A new generation of short-range $(<150 \mathrm{~m})$, low-cost TLS systems provide an affordable option for high-quality TLS data, at the expense of limited range and/or durability. Lower-cost TLS systems are designed primarily for use in surveying, architecture, and civil engineering and remain relatively untested in forestry and ecological applications. Here we compare the utility of two common lower-cost $(<\$ 40,000$ USD) TLS systems for deriving forest structural parameters: 1) The Leica BLK360 - a less durable (e.g. exposed rotating mirror and nonwaterproof) mid-range $(60 \mathrm{~m})$ time-of-flight system; and 2) the Faro Focus 120 3D TLS, a mid-range $(120 \mathrm{~m})$ phase-shift system.

The Leica BLK360 (henceforth Leica) and the Faro Focus 120 3D (henceforth Faro) differ in the method of retrieving range estimates -the Leica is a time-of-flight (TOF) system, while the Faro is a phase-shift (PS) based system. Calders et al [1] describes the difference between time-of-flight and phase-shift systems in detail. Briefly, TOF systems emit discrete light pulses and measure the time it takes for that pulse to return to the sensor. In contrast, PS systems measure the difference in phase between a continuous outgoing and incoming signal, the difference or "shift" in phase determines the range estimate. PS scanners are generally less expensive than time-of-flight systems while having smaller beam divergence (an instrument specification determining laser footprint at distances), but the ranging method results in greater point cloud noise. The Leica examined here is the only time-of-flight system at the time of this study below 40,000 USD.

To compare these two scanners, we first directly assess the point clouds from each instrument by comparing the return distribution as a function of range and height above ground. We augment this by further providing a direct comparison of point cloud noise. Next, we extract and compare tree-level standard forestry measurements (tree diameter and height) and total volume estimates. Finally, we evaluate a range of factors that affect foliage profile estimates, conduct a sensitivity analysis of laser scanner range, scan resolution, and point cloud filtering, then conclude with a comparison of the specific characteristics of each instrument's estimated foliage profiles.

\section{Materials and Methods}

\subsection{Study Area and Data Collection}

Terrestrial laser scans were acquired at two sites in central Virginia, USA with similar vegetation composition--secondary growth mixed forests primarily populated by mature chestnut oak (Quercus montana), white oak (Quercus alba), sweetgum (Liquidambar styraciflua), dogwood (Cornus florida), tulip poplar (Liriodendron tulipifera). Eastern white pine (Pinus strobus) and mountain laurel (Kalmia latifolia) were also minor constituents of the forest. Site 1 was located at Observatory Hill on the grounds of the University of Virginia (38.03, -78.52), where we scanned a 0.1 ha circular plot on April 17 and 20, 2018, just prior to leaf-out, specifically to capture woody tree structure. Site 2 was located at the Pace Estate, a property in holding of the University of Virginia $(37.92,-78.27)$. At site 2, which is approximately $25 \mathrm{~km}$ East of Site 1, we scanned a second plot of the same size during the late growing season (September 10, 2018) to compare vertical foliage profiles. At each plot, we arranged eight, $7.2 \mathrm{~cm}$ diameter white polystyrene spheres on $2 \mathrm{~m}$ fiberglass poles throughout the plot to aid in scan registration.

\subsection{Laser Scanners}

Here, we compare the Faro Focus 120 3D and Leica BLK360 laser scanners. The Leica BLK360 is a 3D terrestrial laser scanner with an integrated spherical and thermal imaging system. The BLK360 is a time-of-flight system operating at $830 \mathrm{~nm}$ wavelength with a 360degree horizontal and 300-degree vertical view, a range of 0.6 to $60 \mathrm{~m}$, a measurement rate of 360,000 points per second, with a ranging accuracy of $4 \mathrm{~mm}$ at $10 \mathrm{~m}$ to $7 \mathrm{~mm}$ at $20 \mathrm{~m}$ distance, respectively. The Leica was set to standard scan quality for a total of 18 million pulses per scan. Time elapsed per scan was approximately 2 minutes. The Faro Focus 120 $3 \mathrm{D}$ phase-shift TLS was set to medium resolution and quality (1/5 resolution; $4 \mathrm{x}$ quality; 
0.044 mrad; 122,000 points per second) for a total of 28.2 million pulses per scan. Time elapsed per scan was approximately 3 minutes.

Table 1. Laser scanner overview of specifications and parameters used in the study.

\begin{tabular}{|c|c|c|}
\hline & Faro Focus 120 3D & Leica BLK 360 \\
\hline Ranging Method & Phase-shift & Time-of-Flight \\
\hline Wavelength (nm) & $905 \mathrm{~nm}$ & $830 \mathrm{~nm}$ \\
\hline Laser Class & Class 3R & Class 1 \\
\hline Beam Divergence & $0.19 \mathrm{mrad}$ & $0.4 \mathrm{mrad}$ \\
\hline Specified Ranging Accuracy & $\pm 2 \mathrm{~mm}$ & 4 mm @10 m / 7 mm @ 20 m \\
\hline Resolution (this study) & $1.768\left(0.044^{\circ}\right)$ & $\sim 1.3 \operatorname{mrad}\left(0.074^{\circ}\right)$ \\
\hline Max resolution & $0.157 \operatorname{mrad}\left(0.009^{\circ}\right)$ & $0.576 \operatorname{mrad}\left(0.033^{\circ}\right)$ \\
\hline Max Measurement Rate & 976,000 per second & 360,000 per second \\
\hline Max Range & $120 \mathrm{~m}$ & $60 \mathrm{~m}$ \\
\hline Scanning Time & $1.5 \mathrm{~min}$ & $3 \mathrm{~min}$ (at high)/1.5 mins (at medium) \\
\hline Temperature Operability & $5^{\circ}-40^{\circ} \mathrm{C}$ & $5-40^{\circ} \mathrm{C}$ \\
\hline Weight & $5 \mathrm{~kg}$ & $1 \mathrm{~kg}$ \\
\hline Full Color & 70 Mpix & 150 Mpix + Thermal \\
\hline Software & Faro SCENE & Cyclone REGISTER 360 \\
\hline
\end{tabular}

\subsection{Scan Acquisition and Registration}

We reduced occlusion from the presence of high-density vegetation by scanning 5 times in a diamond pattern oriented at approximately each cardinal direction to provide sufficient coverage and a standardized sampling scheme $[13,15]$. At times, an additional scan was required for full coverage. This scheme was followed for both scanners. To aid in post-processing scan registration, we placed 6-inch diameter polystyrene spheres atop fiberglass stakes throughout the plot. Multiple scans were digitally registered using the registration points, as described in the following section.

The Faro TLS scans were registered in the Faro SCENE 5.4 software, where registration targets were located and the point clouds aligned (plot registration error $=4.8 \mathrm{~mm}$, $\mathrm{sd}=4.8 \mathrm{~mm}$ ). We removed all returns with intensity lower than 650 (maximum value $=$ 2100; [14]). Filtering low intensity returns reduces noise and ensures gaps are correctly identified for the PAVD analysis. We exported all single scan files in a gridded PTX format. Gridded scan formats like PTX are useful for estimating vegetation area index as gaps are retained in the data and flagged by being shifted to the scan origin $(0,0,0)$. The 
gaps are then easily associated with a specific azimuth or zenith angle because the PTX format is written according to the row and column in the scan grid. The registered point clouds were then filtered with a stray point filter. The stray point filter uses a moving $3 \mathrm{x}$ 3 grid cell window with a $20 \%$ retention and $1 \mathrm{~m}$ distance threshold. Finally, we exported the TLS data as a single merged ASCII file for stem modeling.

The Leica BLK 360 scans were registered using Cyclone REGISTER 360. Registration targets were located within the point clouds of each individual scan (or setup as Cyclone REGISTER 360 refers to scans) and then classified as spherical targets within the software. Scans were then auto-aligned using the align cloud feature (plot registration error $=11.6$ $\mathrm{mm}, \mathrm{sd}=1.6$ ). Cyclone REGISTER 360 provides the user with the option to remove ambiguous points, intensity overloaded points, and mixed-pixel points, though only the mixed-pixel points option provides the user with any control over how the filter is applied (i.e. low, medium and high settings). For this reason, all filters were turned off for this comparison. We exported the single merged, or "bundled" TLS scan in the same gridded PTX format as the Faro scans.

\subsection{Comparisons of TLS Measurements}

\subsubsection{Point cloud quality}

With the fully aligned Faro and Leica point clouds we conducted a series of comparisons. We evaluated the differences in point cloud quality and point density distribution with respect to height and range in the leaf-off scans. To evaluate noise in leaf-off conditions, we calculated the nearest neighbor distance between the two point clouds, highlighting noise points (points detected greater than $8 \mathrm{~cm}$ difference from the Leica point clouds).

\subsubsection{Forestry and QSM Measurements}

We separated and modeled individual trees on each plot using an automated workflow within the CompuTree software [33], described in detail in Stovall et al. [13,15]. The processing took place in four steps: ground point classification and DTM creation, stem identification, tree segmentation, and stem reconstruction with QSMs. The tree was automatically segmented from the point cloud using an iterative nearest neighbor approach, starting at the initial seed point on the stem and moving vertically while expanding in area with the expanding tree crown. On segmented trees, we estimated diameter at breast height (DBH), height, and total volume for 31 trees across the 0.1 ha plots. DBH was estimated at $1.3 \mathrm{~m}$ above ground using cylindrical least squares fitting as part of the SimpleTree quantitative structure modeling (QSM) algorithm $[34,35]$ within the CompuTree software [33]. Two DBH estimates are provided in the software: uncorrected or corrected, based on cylinder fits above and below the $1.3 \mathrm{~m}$ DBH height. We evaluated both DBH estimates. Tree height is also outputted from SimpleTree, as the tallest recorded point above ground for a segmented tree. To evaluate the total tree volume estimates from each TLS, we modeled the segmented tree point clouds using the SimpleTree method [35].

\subsubsection{Foliage Profiles}

PAVD distributions are derived from a simple calculation of the vertically resolved gap probability (Pgap). Pgap is calculated as:

$$
\operatorname{Pgap}(\theta, z)=1-\frac{\left(z_{i}<z, \theta\right)}{N(\theta)}
$$

Where $\mathrm{z}$ is the height above ground and $\theta$ is the midpoint of the 5 degree zenith angle range used to aggregate the LiDAR returns. The equation essentially calculates the cumulative number of returns per unit height divided by the total number of outgoing laser pulses. In essence, as more vegetation is intercepted, the probability of a laser pulse escaping the canopy decreases. 
PAVD is accurately estimated at the hinge angle - a viewing zenith angle of 57.5 degrees - with $P_{g a p}$. The hinge angle is used to estimate total plant area index (PAI) as this is the angle at which the G-Function is nearly invariable at 0.5 for all typical leaf angle distributions [36-38]. A five-degree zenith bin between 55 and 60 degrees is used to approximate the hinge angle region. Pgap is converted to the cumulative PAI distribution with respect to height aboveground $(L(z))$ using:

$$
L(z) \approx-1.1 \log \left(P_{g a p}(57.5)\right)
$$

Estimates of $L(z)$ at the top of the canopy approximate the total PAI for a particular sample location. Once cumulative PAI is estimated, $P_{g a p}$ is calculated at 5-degree zenith bands from 0-60 degrees and weighted by the sin of the zenith angle to sample the uppermost area of the hemisphere viewable by the laser scanner. Finally, the total PAI in each 1 $\mathrm{m}$ vertical bin is estimated as the 1st derivative of the $L(z)$ curve after weighting with respect to zenith angle.

We tested the sensitivity of scanner range, resolution, and gridding on the final PAVD profile. For the first two tests we focused on the Faro scanner, as it had the greatest resolution and range in the study, making it an ideal baseline for evaluating the factors affecting PADV profile estimates. Scanner range was tested at $10 \mathrm{~m}$ intervals, excluding points above the range threshold of interest and then processing the resulting point cloud as above. Scan resolution was tested by decimating the point cloud by a factor of 10, 100, 1,000 , and 10,000. The resulting point clouds were processed as above to produce PAVD and PAI estimates.

The highly filtered unstructured (not retaining the original pulse grid) Leica point clouds produced extreme underestimates of total PAI and PAVD, so we developed a point cloud gridding method useful for deriving PAI and PAVD profiles. The raw Leica data with xyz information is converted into zenith, azimuth, and range using a cartesian to spherical coordinates function. The zenith and azimuth angles can then be projected on a consistent artificial scan grid. In our case, the leica data was collected on a grid of $\sim 2410$ (rows; zenith) $x \sim 4821$ (columns; azimuth). We say approximately because for a single scan the scan grid varies, depending on the stopping point of the laser scanner. From this we determined the scan resolution was $\sim 0.075$ degrees or $1.3 \mathrm{mrad}$. To ensure gaps in the scan data were filled we decreased the scan grid resolution by a factor of 2 to $2.6 \mathrm{mrad}$. Using the rasterize function in the raster package ( $\mathrm{R}$ programming language) we created an artificial scan grid spanning the observed zenith and azimuth range at $2.6 \mathrm{mrad}$ resolution. For a given set of points that fall within a unique zenith and azimuth coordinate range the mean range value is calculated, creating a gridded set of mean TLS range observations. We then re-convert the gridded TLS data from spherical coordinates back to cartesian coordinates, processing using the normal PAI and PAVD processing pipeline described above.

\section{Results}

\subsubsection{Point cloud quality}

In comparing the two scanners, there are clear differences in point cloud quality visually as well as the distribution of observed returns. The Leica point cloud had substantially lower noise than the Faro data. Our comparison of the aligned point clouds in leaf off conditions highlight this issue clearly (Figure 1). The Faro data - though filtered - have obvious ranging errors on the edges of small objects, specifically small branches across a range of distances. In comparison to the Faro, a larger proportion of the returns in the Leica data occur at a closer distance to the scanner. The log scale distribution of returns in the Z-dimension show how the filtered Leica data has no returns above the canopy surface, while the Faro includes extraneous returns in the raw data. Though the Leica scanner is only rated to capture returns up to $60 \mathrm{~m}$, we observed returns as far as $100 \mathrm{~m}$ in our comparisons of the point cloud distributions. 


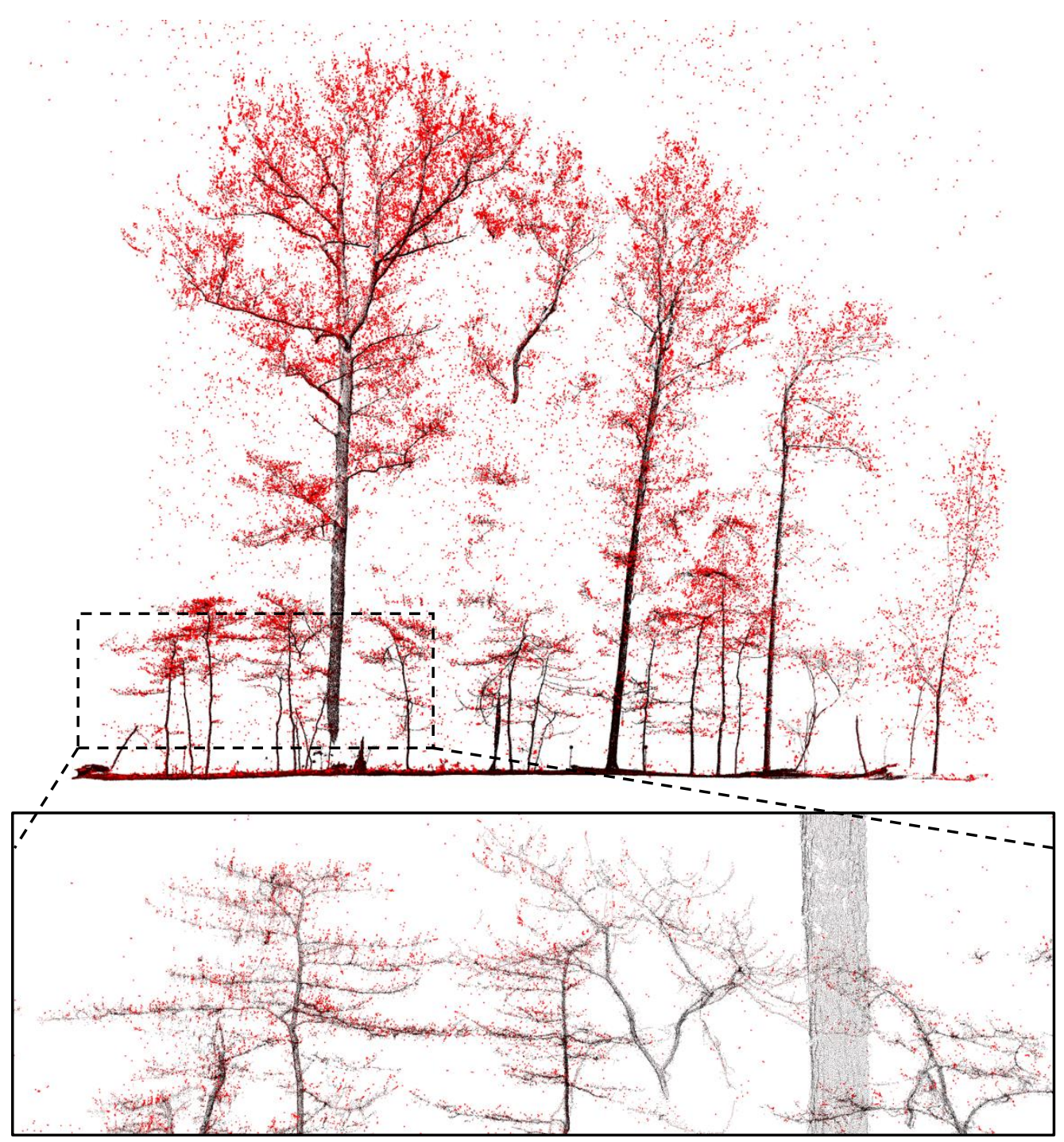

Figure 1. Cross-section of Faro point cloud compared to Leica with noise points highlighted (red). A point was classified as noise if the distance to the Leica point cloud was greater than $8 \mathrm{~cm}$ point distances below $8 \mathrm{~cm}$ were assumed to be registration error. 

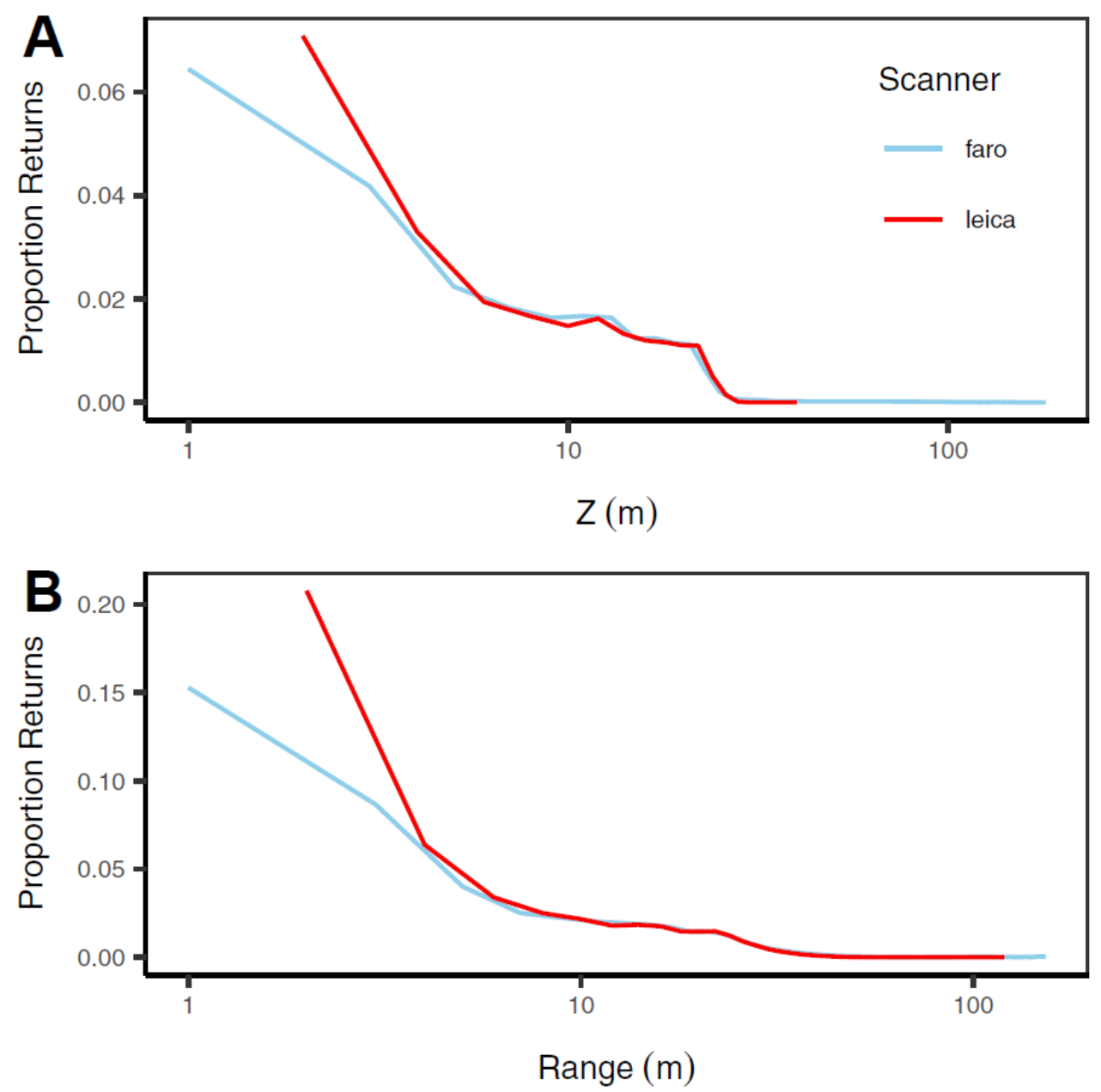

Figure 2. Comparison of the density of Faro and Leica returns in the $[\mathrm{A}]$ vertical and $[\mathrm{B}]$ horizontal dimensions.

\subsubsection{Forestry and QSM Measurements}

Tree diameter and height were in close agreement across scanners (Figure 3; Table 2), with an RMSE of 3.8-4.0 (16-17\%) for DBH, with the Faro estimating smaller values by $\sim 6-10 \%$. Tree height estimates had an RMSE of $1.1 \mathrm{~m}$ or $6.0 \%$ but were virtually unbiased. Volumetric estimates differed greatly between scanners, with the Leica having an average of $\sim 0.31$ or $\sim 29 \%$ higher volume than the Faro QSMs. RMSE was $\sim 0.6 \mathrm{~m}^{3}$ or $56 \%$. Through visual inspection, we highlight key areas of the point cloud and modeling where dramatic differences occur (Figure 4).

Table 2: Statistics comparing the two laser scanners. The bias estimates were relative to the Faro estimates. Parentheses in volume comparison show statistics excluding the largest modeled tree.

\begin{tabular}{ccccc} 
& RMSE & RMSE $(\%)$ & Bias & Bias (\%) \\
\hline DBH $(\mathrm{cm})$ & 3.83 & 16.01 & 1.07 & 4.46 \\
${ }^{*}$ DBH $(\mathrm{cm})$ & 4.03 & 17.33 & 1.32 & 5.69 \\
Height $(\mathrm{m})$ & 1.07 & 6.01 & 0.25 & 1.38 \\
Volume $\left(\mathrm{m}^{3}\right)$ & 0.61 & 56.33 & 0.31 & 28.97 \\
\hline
\end{tabular}



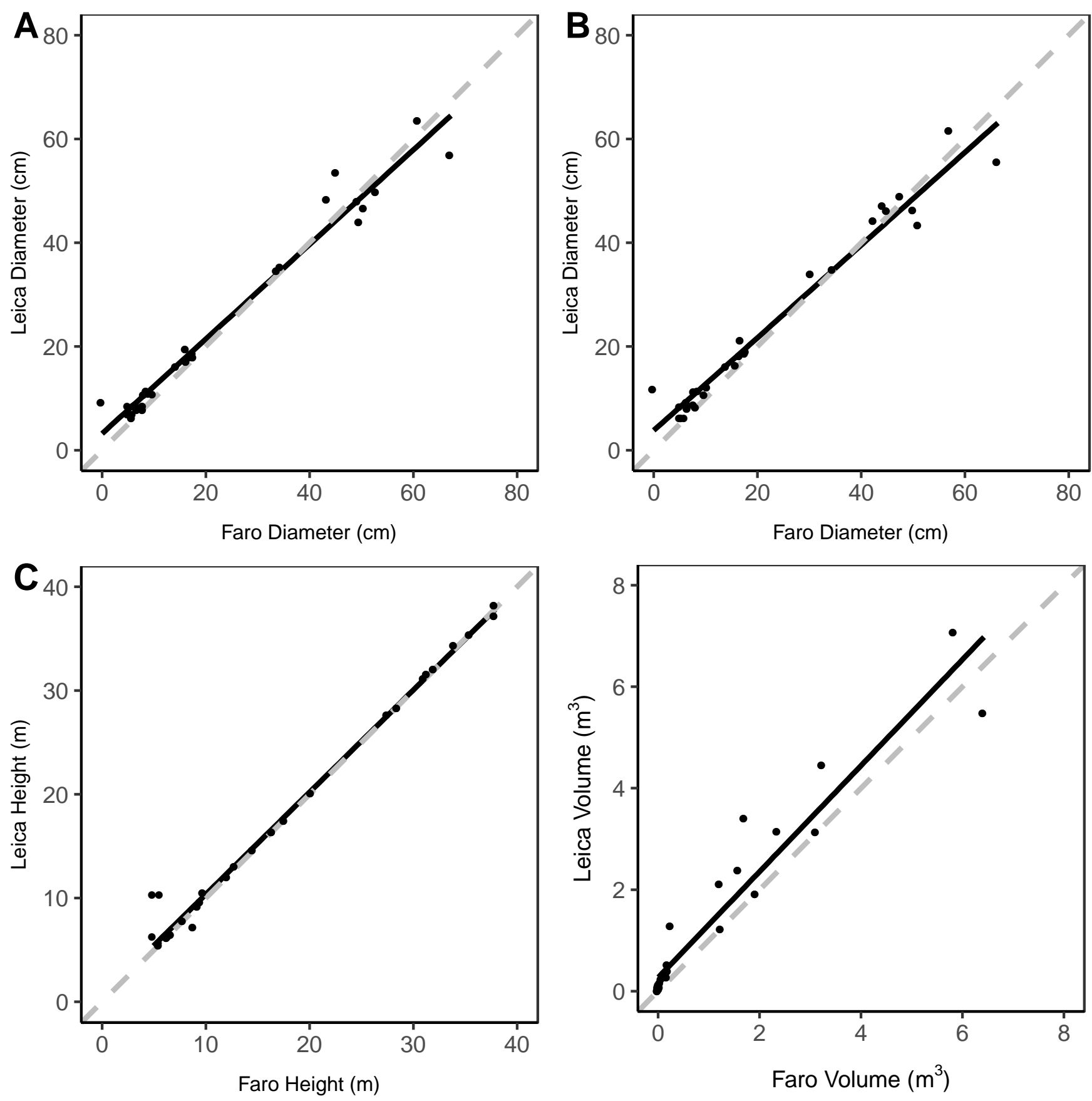

Figure 3. Comparison of [A] uncorrected DBH, [B] corrected $\mathrm{DBH},[\mathrm{C}]$ height, and [D] total tree volume estimated from Faro and Leica laser scanners. 
Faro


Leica
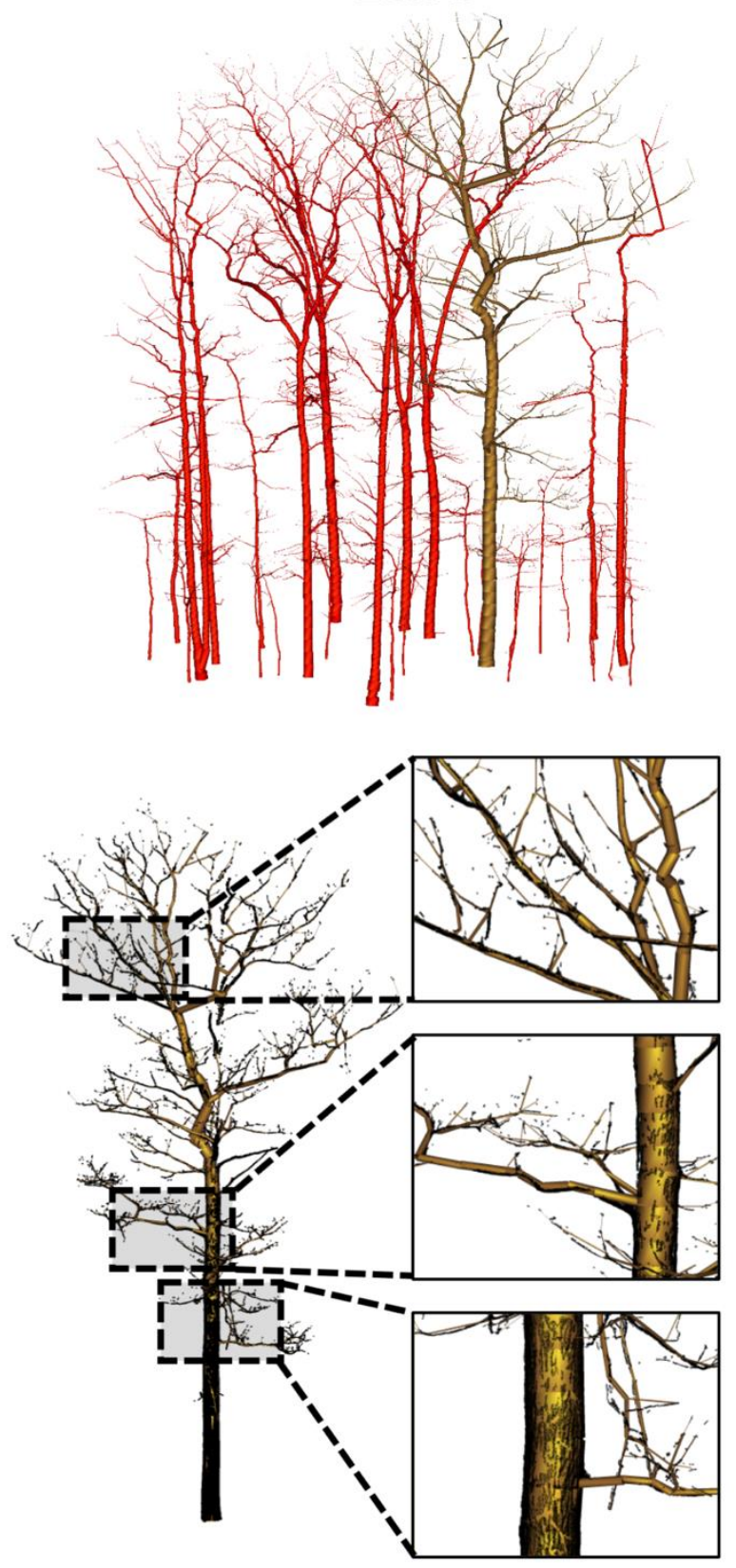

Figure 4. [top] Overview of the resulting TLS QSMs for Faro (blue) and Leica (red) with subset tree (brown) shown in detail. The QSMs in comparison to the point cloud (black) show marked differences depending on instrument, with Faro point clouds having several inaccurate fits and Leica closely matching point cloud structure. 


\subsubsection{Foliage Profiles}

PAVD profiles were insensitive to maximum range until $\sim 45 \mathrm{~m}$ - the point where canopy returns nearest the scanner are lost. Below this, PAVD profiles reduce in height and total PAI decreases substantially (from $\sim 2$ to less than 0.5 ). Point cloud or laser scanner resolution had a minimal effect on the overall shape of the PAVD profile. Higher degradation of scanner resolution resulted in higher variability in the profile reconstructions, but the overall shape remained consistent. The same was true of the PAI estimates, with more degraded laser scans resulting in higher PAI variability. Gridding the Leica point cloud resulted in a closely matched PAVD profile, similar to the Faro. RMSE of the gridded PAVD profile was $0.0153 \mathrm{~m} 2 \mathrm{~m}-2$ or $\sim 25 \%$ and was virtually unbiased $(0.8 \%)$. In contrast, the ungridded Leica data had large errors in the PAVD profile $(\mathrm{RMSE}=100 \%$; Bias $=-91 \%$ ).
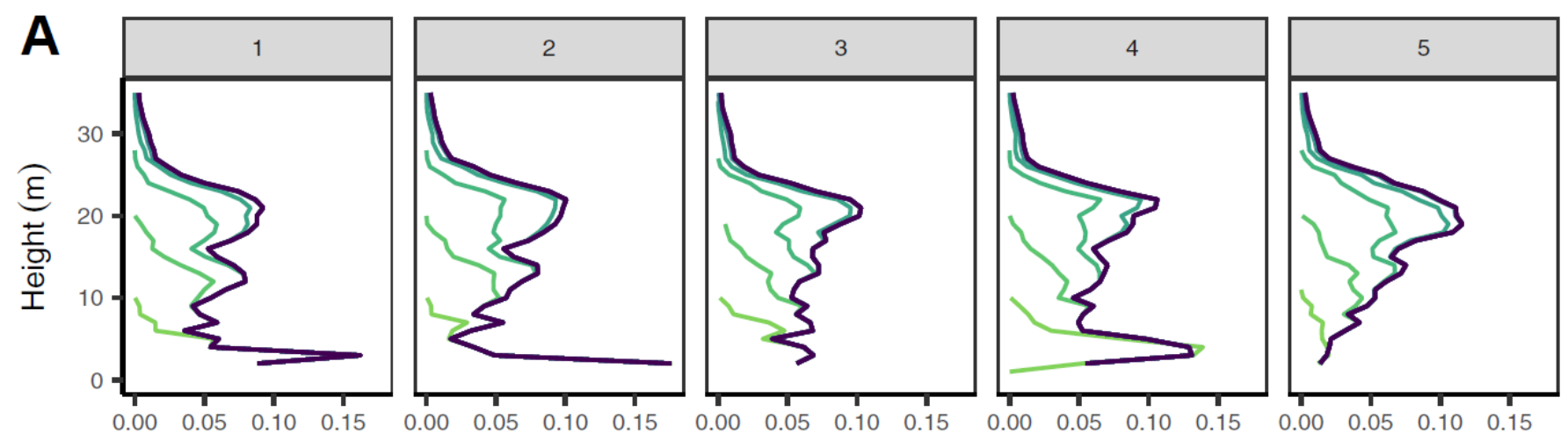

$\operatorname{PAVD}\left(\mathrm{m}^{2} \mathrm{~m}^{-2}\right)$

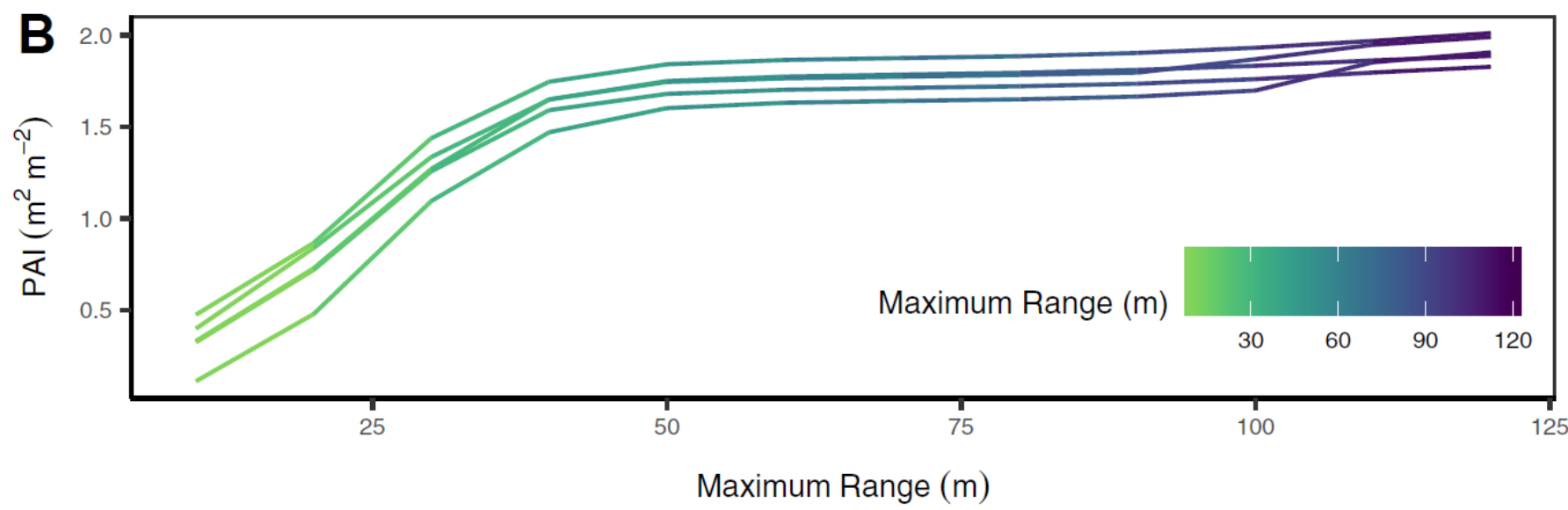

Figure 5. Effect of reducing maximum range on [A] vegetation profiles and [B] total Plant Area Index (PAI) at 5 scan locations using the Faro laser scanner. Reducing the maximum range reduces estimates of foliage profiles and PAI if the laser is unable to exit the canopy. Here, a $\sim 30 \mathrm{~m}$ canopy requires approximately $50 \mathrm{~m}$ maximum range to provide unbiased estimates of PAI. 

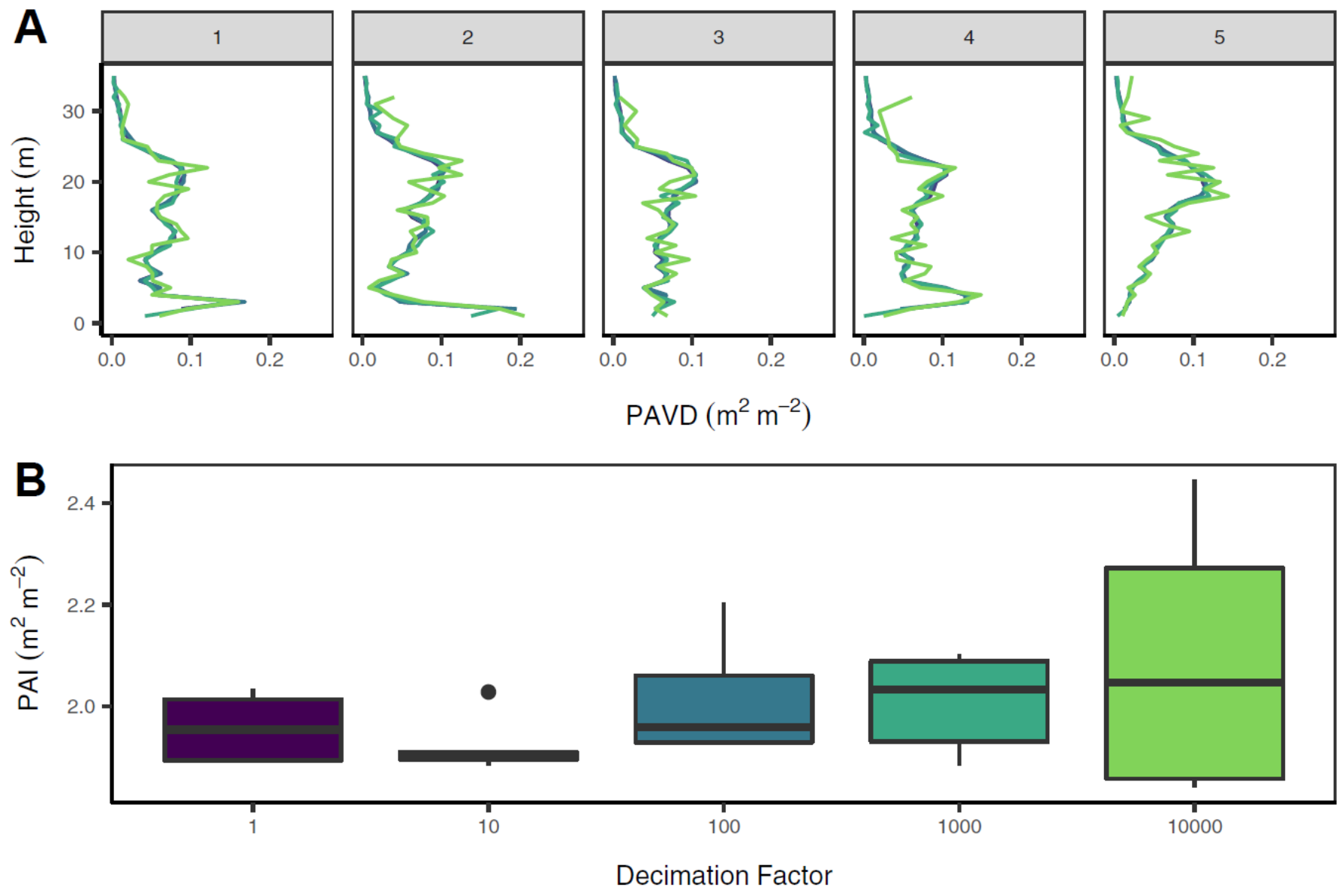

Figure 6. Effect of point cloud resolution on [A] vegetation profiles and [B] total Plant Area Index (PAI) at the single scan level. Reducing point cloud resolution by decimation has little effect on the magnitude of foliage profiles and PAI, but increases variability across scans. Foliage profiles stayed stable up to a decimation factor of 10,000 . As little as $~ 3000$ points per scan can provide reliable estimates of foliage profiles, at the expense of increased variability in PAI estimates. 

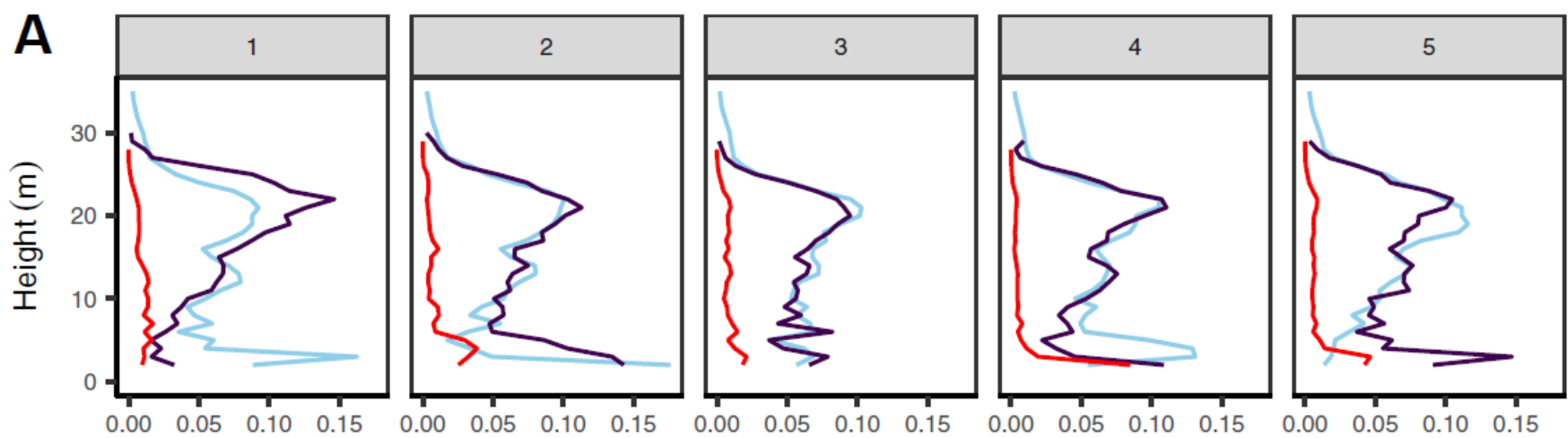

$$
\operatorname{PAVD}\left(\mathrm{m}^{2} \mathrm{~m}^{-2}\right)
$$

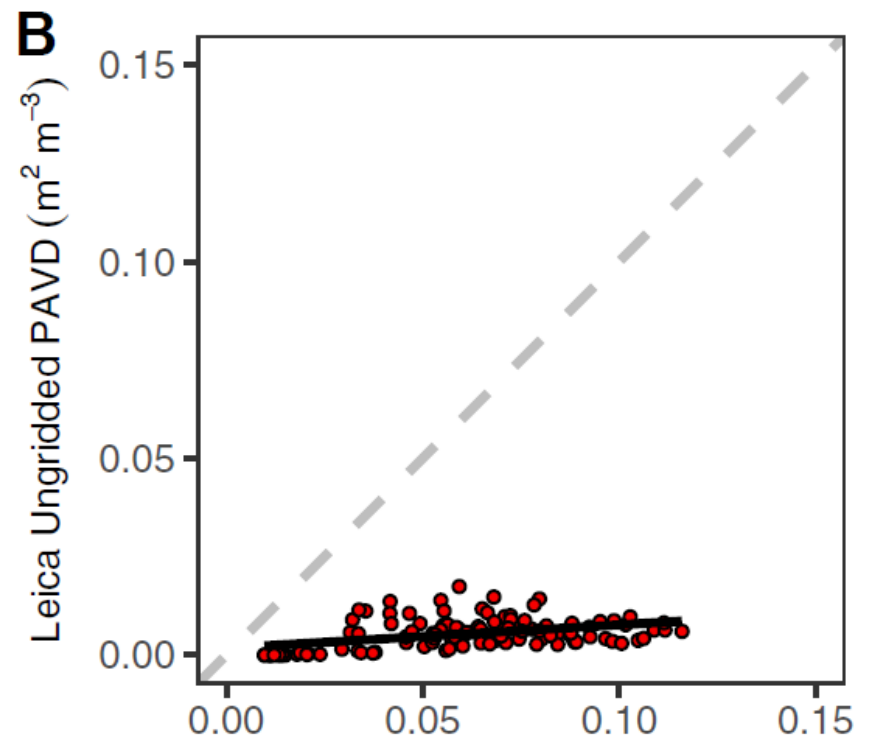

Faro PAVD $\left(\mathrm{m}^{2} \mathrm{~m}^{-3}\right)$
C

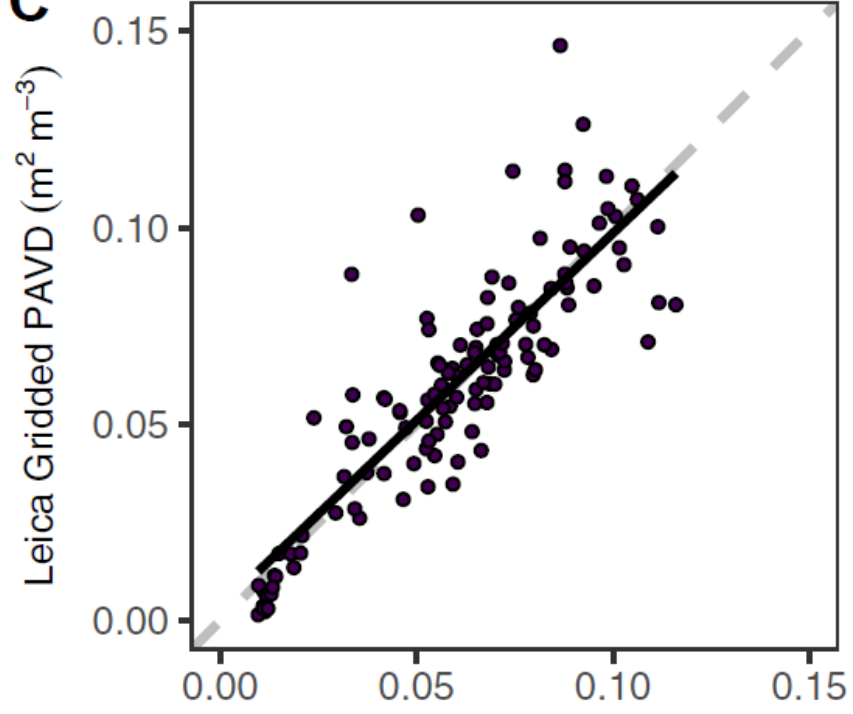

Faro PAVD $\left(\mathrm{m}^{2} \mathrm{~m}^{-3}\right)$

Figure 7. [A] Gridded (purple) and raw filtered (red) Leica point cloud in comparison to Faro (blue) for estimates of foliage profiles. [B] The ungridded Leica point cloud (red) consistently underestimates PAVD. [C] Gridding the Leica data makes PAVD estimates comparable to the Faro data. 


\section{Discussion}

We compared the ability of two low-cost terrestrial laser scanners to detect forest structural attributes. We assessed the point clouds from each instrument by comparing the return distribution and evaluating point cloud noise, clearly showing reduced noise in the filtered data from the Leica instrument. We further compare extracted tree-level diameter and height, showing consistent estimates with less than $1-5 \%$ bias. Volumetric estimates from QSMs were less consistent, with $29 \%$ bias, depending on model reconstruction quality. Finally, our sensitivity analysis of factors affecting foliage profile estimates underscored the need for scanners with appropriate range that retain all pulse information. Surprisingly, scan resolution of extremely degraded quality produced unbiased, but variable PAI estimates. The factors evaluated in this comparison point towards necessary considerations for future low-cost laser scanner development and application in detecting forest structural parameters.

\subsection{Point Cloud Quality}

The quality of TLS point clouds is affected by a suite of instrument parameters/specification and field conditions - all of which affect the products and estimates derived [1]. The technology used to make ranging estimates with TLS can be phase-shift (e.g. Faro) or time-of-flight (e.g. Leica). Phase shift instruments are known to offer fast, high-resolution scans, but suffer from ranging errors for partial interceptions, such as small branches or leaves and edges of larger trunks (Figure 1). In contrast, time-of-flight TLS instruments are typically less sensitive to these edge errors, producing low-noise point clouds, but do not provide the same level of detail found in phase-shift TLS data. All currently available time-of-flight TLS have larger beam divergence than their phase-shift counterparts, consistent with the scanners in this study. Beam divergence of an instrument is the main technical limitation for many laser scanners, since this controls the size of the laser footprint at distance. Larger beam divergences of time-of-flight sensors may result in an inability to detect small gaps in the canopy [39]. In leaf-off conditions, however, this large beam divergence is less problematic for detection of interceptions but presents issues with accurate estimates of small branch size. Here, in the winter scans, we found the Leica to produce a much cleaner (i.e., less noise) point cloud than the Faro and, while the Leica range was lower, the overall quality at high distances was superior (Figure 1 and 2). This was particularly obvious in the presence of anomalous returns for the Faro data, where noise points could be found far above the forest canopy. These noise points are nearly unavoidable, as they result from interference from solar radiation and inaccurate range estimates on the edges of objects. Filtering to the extent of removing this noise severely impacts the detail in point clouds, so a balance must be struck between filter parameters and presence of noise in these phase-shift data. In terms of point cloud quality with minimal user intervention, we conclude that the Leica provides acceptable data for single tree extraction and individual tree modeling, assuming appropriate quality control is implemented during post-processing.

\subsection{Forestry and QSM Measurements}

The fundamental basis of forest measurement lies in consistent plot-based measurements of inventory variables, such as diameter at breast height (DBH) and tree height. Here, we found agreement across sensors on the order of typical human error, with $\sim 4 \mathrm{~cm}$ RMSE and $1 \mathrm{~m}$ for diameter and height, respectively. Since diameters were derived from a fully automated tree modeling process (SimpleTree) we expect even better agreement with algorithms optimized specifically for diameter measurement (e.g. past studies have sub-centimeter error [14]). Similarly, tree height estimates were derived directly from the derived tree-level QSMs, so point cloud approaches to height and diameter estimation would have likely provided much higher precision across sensors [14]. As such, we believe measurements such as diameter and height are comparable enough for studies that 
wish to incorporate the two scanners, with the expectation that a small validation plot of overlapping scans is collected to provide a reference baseline for sensor agreement.

Point cloud quality was the major factor affecting QSM-based volumetric estimates. Our detailed comparison of QSM fitting highlights how small errors in cylinder fits can result in large errors in total volume (Figure 3 and Figure 4). In general, it is clear from visual inspection that different representations in the small branches and noise levels directly affect the QSM reconstruction quality. Leica estimated much higher tree volumes, on average, but we believe the estimates to be more accurate than Faro, since many errors in cylinder fits were apparent (Figure 4). The inconsistency of the two sensors with respect to QSM creation points to a larger issue with TLS measurements as a whole - different sensors can produce inconsistent data quality that ultimately propagates error and uncertainty to tree-level products [40]. In our study we ensured the scan position was virtually identical (within a few centimeters), but with differing scan acquisition strategies we expect variation in upper canopy occlusion [41] will result in dramatic differences in QSM creation. This finding points to the need for consistent acquisition strategies that are optimized to minimize occlusion - only strategies that reduce occlusion will provide the most consistent estimates of tree-level volume and biomass.

There is a clear need for objective means of evaluating point cloud and QSM quality. In Figure 8 we show our proposed method of providing a simple index of QSM fit quality. The basic method requires a simple distancing of the point cloud to the QSM cylinder mesh. Visualization of the residual point cloud distances (shown here as a divergent redwhite-blue gradient) enables easy quality control of QSM creation. A statistical approach using this method could estimate the statistical parameters of the QSM-cloud distances, deriving mean and standard deviation. Here, we show how, in contrast to the Faro QSMfit, the better fit from Leica data provides lower standard deviation and a mean closer to 0 . Objective methods of QSM and point cloud quality, such as these, will provide more consistent estimates from global-scale TLS datasets. 



Figure 8. Proposed method of evaluating QSM and point cloud quality. (top) Poor QSM fits are highlighted in red on the Faro point cloud. The statistics from the distribution of distances to the QSM cylinders (mean and standard deviation) can offer an objective method of evaluating the validity of cylinder fits across tree reconstructions.

\subsection{Foliage Profiles}

The most important instrument specifications and processing settings relevant for reconstructing PAVD profiles relate to range, resolution, and filtering. In our analysis, we found very little effect of range until the $35 \mathrm{~m}$ range threshold since, in a 25-30 m canopy, a 30-35 m range is required to exit the canopy top. As such, most TLS units have a range capable of measuring most canopy heights that exist globally. Of the low-cost TLS units evaluated here, the Faro is capable of measuring canopy structure in the tallest forest (Redwood species; $100 \mathrm{~m}$; see [42] using Riegl VZ400), but the high noise with range makes the quality of such PAVD profiles potentially error prone. The maximum forest canopy 
height that can be measured with the Leica TLS is $\sim 50 \mathrm{~m}$, which covers a large range of existing forest heights, making this particular instrument broadly applicable.

Surprisingly, PAVD profiles were minimally affected by scan resolution, with an increase in profile variability, but the broad shape and trends of the profile reconstructions remained consistent. The implications of these results are applicable to future low-cost TLS unit development. In forest stands with PAI up to $\sim 4-5$, we believe TLS units can be developed with lower angular resolution to reconstruct PAVD profiles. Developments such as this have the potential to reduce data volume and increase portability of instruments to the extent that they can become more ubiquitous in their deployment in field sites globally. One example of a low-cost and automated instrument specifically designed for forests exists (LEAF instrument, [43]. While specifications such as beam divergence may be a hindrance, studies of the sensitivity of parameters, as explored here, are key to capturing the needed technical specifications for forest-specific instruments that become more affordable for the scientific community.

For the heavily filtered unstructured Leica point clouds gridding of TLS scans was necessary to estimate the PAVD profile. The close agreement between Leica and Faro data ( $25 \%$ RMSE) and unbiased estimates are, in part, related to the relative insensitivity of the PAVD profile to scan resolution, as discussed above. However, we have uncovered a potential tradeoff between high quality filtered TLS data and the reconstruction of PAVD profiles. For example, the necessary workflow for creation of QSMs and PAVD profiles is specific to the desired end product. PAVD profiles may require minimum filtering of edge artifacts with a particular focus on clear quantification of gaps in the canopy. In contrast, QSM creation requires extremely clean, denoised point clouds to provide reliable results. As it stands, these issues remain unresolved in the TLS community and the need for a standardized and systematic processing workflow is necessary to ensure consistency in data quality as TLS is more widely adopted.

\subsection{Workflow Considerations and Recommendations}

Outside of the specific parameters tested in this study, there are marked differences in the field and pre-processing workflow steps between instruments, affecting the efficiency of the entire pipeline of TLS processing. Since TLS requires field-based data collection, one major consideration in all instruments is ease of use in this setting. With several years of experience using the Faro instrument, the authors found field-based data collection to be efficient and without unexpected delays. Rarely, the Faro instrument would be unable to operate due to temperatures outside of the safe specified range and in some instances instrument startup would fail; Though all situations resolved in the field and did not disrupt data collection. The availability of onboard touchscreen and removable storage space is key to ensuring data collection was successful - often revisiting a field plot may be impossible, so verifying data was collected successfully is essential.

The authors found the Leica to also be efficient in field-based data collection--with scan acquisition times of similar magnitude to the Faro and with the added benefit of lighter weight, and smaller stature making moving from plot to plot easier. The Leica has a more restrictive temperature operating range than the Faro and does not scan below $10^{\circ}$ C. A scanning campaign in central Indiana, USA had to be aborted by the authors in 2019 simply for this reason as unseasonably cool, spring temperatures prevented scanning. The long-term reliability and durability of the Leica is potentially endangered by the lack of cover or protection for the scanning mirror and the construction of the proprietary tripod. While the Leica tripod is lightweight, this comes at the cost of durability as the tripod is not constructed to the standards required for rigorous sustained field use. Additionally, the Leica tripod has a specific adapter that fits the Leica, restricting the use of any other tripod without aftermarket modifications. The Leica also stores data internally, on a $50 \mathrm{gb}$ solid state drive which can only be accessed via a Wi-Fi connection directly with the unit. Via a download management software interface, TLS scan files can then be downloaded in a proprietary format that must be opened with a Leica brand or related software (Cyclone, Autodesk, etc.). Further Autodesk programs such as Recap Pro which can be used 
directly with the scanner in the field via an iPad wifi connection, automatically filter out points in a manner that the user cannot control (for which we specifically developed the PAVD gridding technique). While this method may be suitable for construction or architectural work where use-cases may involve hard edges and angles, this is not adequate for forestry or any other ecological related work in a natural environment. The information loss is far too great, and the resulting data are not suitable. For example, the entire leaves and small branches appear to be removed from scans when this acquisition method is used. The only exception to this may be work quantifying microtopography [9], yet we advise strongly against the iPad-Autodesk/ReCap pipeline. Additionally, downloading files from the Leica takes several minutes per file, as does importing data into Cyclone.

In summary, we found these two low-cost laser scanners were comparable, but key differences in data quality have led us to several recommendations for users of these instruments. [i] The two instruments effectively and comparably estimate field inventory forestry measurements. We believe the simplicity of such measurements make most laser scanners capable of estimating these parameters and future adoption of TLS for this purpose is likely at an operational stage. [ii] QSM reconstruction is highly dependent on point cloud quality and thus will be directly impacted by laser scanner choice. In our case the higher-noise phase-shift data was problematic, resulting in less certain QSM cylinder fits, while low-noise time-of-flight data is best, with the expectation of potentially overestimating small branch volume due to larger beam divergence. [iii] PAVD profiles with the highest precision require laser scanners that retain the laser pulse grid, but artificially reconstructing gridded data can provide comparable PAVD profile results. While range limits on TLS instruments potentially present problems in tall forest canopies, most stationary laser scanners capture the range necessary to measure PAVD profiles in forest greater than $50 \mathrm{~m}$ in stature. All recommendations do not point to a clear choice for the ideal laser scanner. Most compromises with the Leica instrument are in ease of workflow and field data collection, along with the lack of retained pulse grid information. The Faro instrument was efficient to use in the field and - due to the ranging technology - had far noisier point clouds, directly affecting the consistency of QSMs, but PAVD profiles could be reconstructed at a high resolution. In essence, without an available laser scanner that satisfies all requirements specific to measurement of forest structure, the TLS community must quantify the uncertainty introduced by a particular suite of factors that directly affect point cloud quality and PAVD profile reconstruction; Only then can the diversity of TLS instruments (of all price points) be aggregated to confidently address global-scale hypotheses related to forest structure.

Author Contributions: A.S. and J.A. conceived of the manuscript. A.S. and J.A. collected and preprocessed the TLS data. A.S. conducted the analyses. A.S. and J.A. wrote and edited the manuscript.

Conflicts of Interest: The authors declare no conflict of interest.

\section{References}

1. Calders, K.; Adams, J.; Armston, J.; Bartholomeus, H.; Bauwens, S.; Bentley, L.P.; Chave, J.; Danson, F.M.; Demol, M.; Disney, M.; et al. Terrestrial Laser Scanning in Forest Ecology: Expanding the Horizon. Remote Sensing of Environment 2020, 251, 112102, doi:10.1016/j.rse.2020.112102.

2. Lau, A.; Martius, C.; Bartholomeus, H.; Shenkin, A.; Jackson, T.; Malhi, Y.; Herold, M.; Bentley, L.P. Estimating ArchitectureBased Metabolic Scaling Exponents of Tropical Trees Using Terrestrial LiDAR and 3D Modelling. Forest Ecology and Management 2019, 439, 132-145, doi:10.1016/j.foreco.2019.02.019.

3. Stovall, A.E.L.; Masters, B.; Fatoyinbo, L.; Yang, X. TLSLeAF: Automatic Leaf Angle Estimates from Single-Scan Terrestrial Laser Scanning. New Phytologist 2021, n/a, doi:https://doi.org/10.1111/nph.17548. 
4. Vicari, M.B.; Pisek, J.; Disney, M. New Estimates of Leaf Angle Distribution from Terrestrial LiDAR: Comparison with Measured and Modelled Estimates from Nine Broadleaf Tree Species. Agricultural and Forest Meteorology 2019, 264, 322-333, doi:10.1016/j.agrformet.2018.10.021.

5. Ashcroft, M.B.; Gollan, J.R.; Ramp, D. Creating Vegetation Density Profiles for a Diverse Range of Ecological Habitats Using Terrestrial Laser Scanning. METHODS IN ECOLOGY AND EVOLUTION 2014, 5, 263-272, doi:10.1111/2041-210X.12157.

6. Loudermilk, E.L.; Hiers, J.K.; O’Brien, J.J.; Mitchell, R.J.; Singhania, A.; Fernandez, J.C.; Cropper, W.P.; Slatton, K.C. GroundBased LIDAR: A Novel Approach to Quantify Fine-Scale Fuelbed Characteristics. International Journal of Wildland Fire 2009, 18, 676-685, doi:10.1071/WF07138.

7. Hudak, A.T.; Evans, J.S.; Stuart Smith, A.M. LiDAR Utility for Natural Resource Managers. Remote Sensing 2009, 1, 934-951, doi:10.3390/rs1040934.

8. Chen, Y.; Zhu, X.; Yebra, M.; Harris, S.; Tapper, N. Development of a Predictive Model for Estimating Forest Surface Fuel Load in Australian Eucalypt Forests with LiDAR Data. Environmental Modelling E Software 2017, 97, 61-71, doi:https://doi.org/10.1016/j.envsoft.2017.07.007.

9. Stovall, A.E.L.; Diamond, J.S.; Slesak, R.A.; McLaughlin, D.L.; Shugart, H. Quantifying Wetland Microtopography with Terrestrial Laser Scanning. Remote Sensing of Environment 2019, 232, 111271, doi:https://doi.org/10.1016/j.rse.2019.111271.

10. Diamond, J.S.; McLaughlin, D.L.; Slesak, R.A.; Stovall, A. Pattern and Structure of Microtopography Implies Autogenic Origins in Forested Wetlands. Hydrology and Earth System Sciences 2019, 23, 5069-5088, doi:10.5194/hess-23-5069-2019.

11. Walter, J.A.; Stovall, A.E.L.; Atkins, J.W. Vegetation Structural Complexity and Biodiversity in the Great Smoky Mountains. Ecosphere 2021, 12, doi:10.1002/ecs2.3390.

12. Diamond, J.S.; McLaughlin, D.L.; Slesak, R.A.; Stovall, A. Microtopography Is a Fundamental Organizing Structure of Vegetation and Soil Chemistry in Black Ash Wetlands. Biogeosciences 2020, 17, 901-915, doi:10.5194/bg-17-901-2020.

13. Stovall, A.E.L.; Shugart, H.H. Improved Biomass Calibration and Validation With Terrestrial LiDAR: Implications for Future LiDAR and SAR Missions. IEEE Journal of Selected Topics in Applied Earth Observations and Remote Sensing 2018, 11, 3527-3537, doi:10.1109/JSTARS.2018.2803110.

14. Stovall, A.E.L.; Vorster, A.G.; Anderson, R.S.; Evangelista, P.H.; Shugart, H.H. Non-Destructive Aboveground Biomass Estimation of Coniferous Trees Using Terrestrial LiDAR. Remote Sensing of Environment 2017, 200, 31-42, doi:10.1016/j.rse.2017.08.013.

15. Stovall, A.E.L.; Anderson-Teixeira, K.J.; Shugart, H.H. Assessing Terrestrial Laser Scanning for Developing Non-Destructive Biomass Allometry. Forest Ecology and Management 2018, 427, 217-229, doi:10.1016/j.foreco.2018.06.004.

16. Calders, K.; Newnham, G.; Burt, A.; Murphy, S.; Raumonen, P.; Herold, M.; Culvenor, D.; Avitabile, V.; Disney, M.; Armston, J.; et al. Nondestructive Estimates of Above-Ground Biomass Using Terrestrial Laser Scanning. Methods in Ecology and Evolution 2015, 6, 198-208, doi:10.1111/2041-210X.12301.

17. Lau, A.; Calders, K.; Bartholomeus, H.; Martius, C.; Raumonen, P.; Herold, M.; Vicari, M.; Sukhdeo, H.; Singh, J.; Goodman, R.C. Tree Biomass Equations from Terrestrial LiDAR: A Case Study in Guyana. Forests 2019, 10, 527, doi:10.3390/f10060527.

18. Momo Takoudjou, S.; Ploton, P.; Sonké, B.; Hackenberg, J.; Griffon, S.; de Coligny, F.; Kamdem, N.G.; Libalah, M.; Mofack, G.I.; Le Moguédec, G.; et al. Using Terrestrial Laser Scanning Data to Estimate Large Tropical Trees Biomass and Calibrate Allometric Models: A Comparison with Traditional Destructive Approach. Methods in Ecology and Evolution n/a-n/a, doi:10.1111/2041-210X.12933.

19. Bi, H.Q.; Turner, J.; Lambert, M.J. Additive Biomass Equations for Native Eucalypt Forest Trees of Temperate Australia UR -://WOS:000222943100012Ｈttp://Download.Springer.Com/Static/Pdf/888/Art\%253A10.1007\%252Fs00468-004-0333z.Pdf?OriginUrl=http\%3A\%2F\%2Flink.Springer.Com\%2Farticle\%2F10.1007\%2Fs00468-004-0333-

Z\&token2=exp=1448246138 acl=\%2Fstatic\%2Fpdf\%2F888\%2Fart\%25253A10.1007\%25252Fs00468-004-0333-

z.Pdf\%3ForiginUrl\%3Dhttp\%253A\%252F\%252Flink.Springer.Com\%252Farticle\%252F10.1007\%252Fs00468-004-0333- 
$\mathrm{Z}^{*}$ hmac=dc5317c0e5d26791872850f8f056e519da7d5773aaec00fa6304ff3b180b5ca4. Trees-Structure and Function 2004, 18, 467479, doi:10.1007/s00468-004-0333-z.

20. Chave, J.; Réjou-Méchain, M.; Búrquez, A.; Chidumayo, E.; Colgan, M.S.; Delitti, W.B.; Duque, A.; Eid, T.; Fearnside, P.M.; Goodman, R.C.; et al. Improved Allometric Models to Estimate the Aboveground Biomass of Tropical Trees. Global change biology 2014, 20, 3177-3190.

21. Mitchard, E.T.A.; Feldpausch, T.R.; Brienen, R.J.W.; Lopez-Gonzalez, G.; Monteagudo, A.; Baker, T.R.; Lewis, S.L.; Lloyd, J.; Quesada, C.A.; Gloor, M.; et al. Markedly Divergent Estimates of Amazon Forest Carbon Density from Ground Plots and Satellites. Global Ecology and Biogeography 2014, 23, 935-946, doi:10.1111/geb.12168.

22. Brown, S.; Schroeder, P.; Kern, J.S. Spatial Distribution of Biomass in Forests of the Eastern USA. Forest Ecology and Management 1999, 123, 81-90, doi:10.1016/S0378-1127(99)00017-1.

23. Jenkins, C.J.; Chojnacky, D.C.; Heath, L.S.; Birdsey, R.A. National-Scale Biomass Estimators for United States Tree Species. For Sci 2003, 49.

24. Chojnacky, D.C.; Heath, L.S.; Jenkins, J.C. Updated Generalized Biomass Equations for North American Tree Species. Forestry 2014, 87, 129-151.

25. Vorster, A.G.; Evangelista, P.H.; Stovall, A.E.L.; Ex, S. Variability and Uncertainty in Forest Biomass Estimates from the Tree to Landscape Scale: The Role of Allometric Equations. Carbon Balance and Management 2020, 15, doi:10.1186/s13021-020-001436.

26. Chave, J.; Condit, R.; Aguilar, S.; Hernandez, A.; Lao, S.; Perez, R. Error Propagation and Scaling for Tropical Forest Biomass Estimates. Philosophical Transactions of the Royal Society B: Biological Sciences 2004, 359, 409-420.

27. Houghton, R.A.; Hall, F.; Goetz, S.J. Importance of Biomass in the Global Carbon Cycle. Journal of Geophysical ResearchBiogeosciences 2009, 114, G00E03, doi:10.1029/2009JG000935.

28. Land Product Validation Subgroup (Working Group on Calibration and Validation, Committee on Earth Observation Satellites) Aboveground Woody Biomass Product Validation Good Practices Protocol. 2021, doi:10.5067/DOC/CEOSWGCV/LPV/AGB.001.

29. Fahey, R.T.; Fotis, A.T.; Woods, K.D. Quantifying Canopy Complexity and Effects on Productivity and Resilience in LateSuccessional Hemlock-Hardwood Forests. Ecological Applications 2015, 25, 834-847, doi:10.1890/14-1012.1.

30. Shiklomanov, A.N.; Bradley, B.A.; Dahlin, K.M.; Fox, A.M.; Gough, C.M.; Hoffman, F.M.; Middleton, E.M.; Serbin, S.P.; Smallman, L.; Smith, W.K. Enhancing Global Change Experiments through Integration of Remote-Sensing Techniques. Frontiers in Ecology and the Environment 2019, 17, 215-224, doi:10.1002/fee.2031.

31. Atkins, J.W.; Bohrer, G.; Fahey, R.T.; Hardiman, B.S.; Morin, T.H.; Stovall, A.E.L.; Zimmerman, N.; Gough, C.M. Quantifying Vegetation and Canopy Structural Complexity from Terrestrial LiDAR Data Using the Forestr Package. Methods in Ecology and Evolution 2018, doi:10.1111/2041-210X.13061.

32. Fahey, R.T.; Atkins, J.W.; Gough, C.M.; Hardiman, B.S.; Nave, L.E.; Tallant, J.M.; Nadehoffer, K.J.; Vogel, C.; Scheuermann, C.M.; Stuart-Haëntjens, E.; et al. Defining a Spectrum of Integrative Trait-based Vegetation Canopy Structural Types. Ecol Lett 2019, 22, 2049-2059, doi:10.1111/ele.13388.

33. Othmani, A.; Piboule, A.; Krebs, M.; Stolz, C.; Voon, L.L.Y. Towards Automated and Operational Forest Inventories with TLidar. In Proceedings of the 11th International Conference on LiDAR Applications for Assessing Forest Ecosystems (SilviLaser 2011); 2011.

34. Raumonen, P.; Kaasalainen, M.; Åkerblom, M.; Kaasalainen, S.; Kaartinen, H.; Vastaranta, M.; Holopainen, M.; Disney, M.; Lewis, P. Fast Automatic Precision Tree Models from Terrestrial Laser Scanner Data. Remote Sensing 2013, 5, 491-520, doi:10.3390/rs5020491.

35. Hackenberg, J.; Spiecker, H.; Calders, K.; Disney, M.; Raumonen, P. SimpleTree-An Efficient Open Source Tool to Build Tree Models from TLS Clouds. Forests 2015, 6, 4245-4294, doi:10.3390/f6114245.

36. MacArthur, R.H.; Horn, H.S. Foliage Profile by Vertical Measurements. Ecology 1969, 50, 802, doi:10.2307/1933693. 
37. Jupp, D.L.B.; Culvenor, D.S.; Lovell, J.L.; Newnham, G.J.; Strahler, A.H.; Woodcock, C.E. Estimating Forest LAI Profiles and Structural Parameters Using a Ground-Based Laser Called 'Echidna®. Tree Physiology 2008, 29, 171-181, doi:10.1093/treephys/tpn022.

38. Calders, K.; Armston, J.; Newnham, G.; Herold, M.; Goodwin, N. Implications of Sensor Configuration and Topography on Vertical Plant Profiles Derived from Terrestrial LiDAR. Agricultural and Forest Meteorology 2014, 194, 104-117, doi:10.1016/j.agrformet.2014.03.022.

39. Newnham, G. Evaluation of Terrestrial Laser Scanners for Measuring Vegetation Structure. A Comparison of the FARO Focus 3D 120, Leica C10, Leica HDS7000 and Riegl VZ1000. 35.

40. Calders, K.; Disney, M.I.; Armston, J.; Burt, A.; Brede, B.; Origo, N.; Muir, J.; Nightingale, J. Evaluation of the Range Accuracy and the Radiometric Calibration of Multiple Terrestrial Laser Scanning Instruments for Data Interoperability. IEEE Transactions on Geoscience and Remote Sensing 2017, 55, 2716-2724, doi:10.1109/TGRS.2017.2652721.

41. Wilkes, P.; Lau, A.; Disney, M.; Calders, K.; Burt, A.; Gonzalez de?Tanago, J.; Bartholomeus, H.; Brede, B.; Herold, M. Data Acquisition Considerations for Terrestrial Laser Scanning of Forest Plots. Remote Sensing of Environment 2017, 196, 140-153, doi:10.1016/j.rse.2017.04.030.

42. Disney, M.; Burt, A.; Wilkes, P.; Armston, J.; Duncanson, L. New 3D Measurements of Large Redwood Trees for Biomass and Structure. Scientific Reports 2020, 10, doi:10.1038/s41598-020-73733-6.

43. Culvenor, D.; Newnham, G.; Mellor, A.; Sims, N.; Haywood, A. Automated In-Situ Laser Scanner for Monitoring Forest Leaf Area Index. Sensors 2014, 14, 14994-15008, doi:10.3390/s140814994. 